ception, lost. In other words, the sponge remains are precisely those which would be left after a long period of disintegration on the sea-bottom. These remarks apply equally well to remains found in chalk and in flint Clearly, then, these remains suffered no rapid burial. Had the sponges found in flint been entombed by a fall of silica gel, a fow complete and live sponges at least would have been embalmed in the process. It may, indeed, be said that the majority would be in tact, and we should have had a large number of class $a$ so preserved. Yet despite the numbers of flint nodules which must have been examined in the last century, we have nothing to show of class $a$ except for isolated spicules. Nothing could be more decisive.

Frequently, one finds in flint the dictyonine framework of a sponge faintly portrayed in a brown stain, and the connexion between these and similar ironstained impressions in the surrounding chalk is obvious. The replacement of the original silica by iron oxide must have taken place prior to the formation of the flint, showing clearly that that formation was not only subsequent, but also took place a long time after the burial of the sponge-remains.

There is yet one other factor, which also seems to have been completely overlooked. If flint had been formed by the relatively sudden precipitation of silica from solution, we have to explain why the remains of all forms of contemporary life found in it are so poor. Taking the Rhynio chert of Aberdeenshire as an example, in which remains of plants and animals, many of minute size, are so beautifully preserved, we should expect to find much better preservation of the fossils enclosed in flint. The famous Whittaker's three-inch, representing a continuous bed estimated at some 240 square miles in extent, should have embalmed a perfect representation of contemporary benthos. Yet this, and other flint similarly bedded, fails entirely in this respect.

The story of the formation is even yet far from complete, but that that formation was subsequent to the deposition of the chalk, nobody who has worked extensively at both recent and fossil sponges will deny.

Department of Zoology,

British Museum (Nat. Hist.), London, S.W.7.

\section{Absorption Spectra of Crystals at Low Tempera- tures as References in the Measurement of Stellar Velocities.}

The vast work of determining and cataloguing radial velocities has made the slitless spectrograph almost indispensable. A number of stars may then be photographed on the same plate at the same time. Such a method is possible only if the starlight passes through some medium which has sharp absorption lines so that the spectrum of each star has within it the absorption lines for references. A medium having sufficiently sharp lines in the proper spectral region does not seem to have been found. I would suggest the use of the crystals of the rare earths maintained at the temperature of liquid nitrogen. Both Dr. Joy, of the Mount Wilson Observatory, and Dr. Shane, of the University of California, have considered in this connexion the absorption spectra of crystals of gadolinium at room temperature. ${ }^{1}$ The absorption lines occur, however, in an unfavourable region of the spectrum.

The spectrum of the dysprosium ion appears particularly adapted for the purpose. At room temperature its spectrum taken with a three prism Steinheil spectrograph contains some diffuse lines between $4000 \mathrm{~A}$. and $4800 \mathrm{~A}$. At the temperature of liquid nitrogen, the lines are as sharp as those of gases. The crystal studied was dysprosium ethyl sulphate. It is possible, though not easy, to grow this crystal perfectly clear. A few trials would doubtless discover a more amenable salt of dysprosium. The acetate is probably easy to grow. A crystal about $2 \mathrm{~cm} . \times 2 \mathrm{~cm} . \times 0.5 \mathrm{~mm}$. of another rare earth, cerium, was grown almost as clear as glass by evaporating a saturated solution in a desiccator containing concentrated sulphuric acid. Much larger crystals can be obtained when some precautions are taken as in the method of Freed and Spedding.

Because of its constant boiling point, liquid nitrogen is preferable to liquid air, by means of which it may

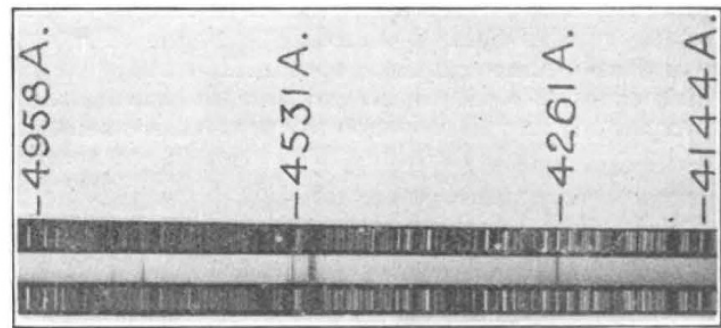

FIG. 1.-Absorption spectrum of dysprosium ethyl sulphate
$\left(\mathrm{Dy}_{8}\left(\mathrm{SO}_{4}\right)_{3} \cdot 9 \mathrm{H}_{2} \mathrm{O}\right)$ at the temperature of liquid nitrogen.

readily be prepared. Also, it has the advantage of complete transparency should one desire to immerse the crystals in the refrigerating liquid.

The high cost of dysprosium might induce one to investigate other rare earths. Neodymium has also been found to possess sharp absorption lines in this spectral region, but they are not so fine as those of dysprosium. However, it is not necessary to employ pure salts of dysprosium. It may be desirable, also for spectrographic reasons, to make a mixed crystal with the corresponding colourless lanthanum salt.

No difficulty should be met in eliminating any serious change in temperature of the spectrograph because of the liquid nitrogen.

I wish to express my indebtedness to Mr. G. P. Kuiper, of the Leyden Observatory, for an interesting conversation. The work is one of a number made possible through the generous hospitality of Prof. W. J. de Haas. The Steinheil spectrograph was kindly placed at my disposal by Prof. W. H. Keesom. Simon Freed

(Fellow of the John Simon Guggenheim Memorial Foundation).

Cryogenic Laboratory,

University of Leyden, Holland, May 23.

1 Freed and Spedding, NaTURE, 123, 525; 1929 : Phys. Rev., 34, $945 ; 1929$.

\section{Mortality among Plants and its Bearing on Natural Selection.}

I HAVE read with great interest the letters from Prof. Salisbury and Dr. J. Phillips which have appeared in NATURE dealing with mortality in plants at different stages of their life histories. The observations are of the greatest importance, but I doubt whether the interpretation advanced by Prof. Salisbury in his letter of May 31, 1930, is supported by the facts. It seems to me that a distinction should be made between mortality and natural selection. The results obtained by Prof. Salisbury with Silene conica $L$. and Verbascum Thapsus $L$. would probably have been very similar if all the seeds in each case had been exactly alike, and, in any case, evolution under natural

$$
\text { No. } 3218 \text {, VoL. 128] }
$$

